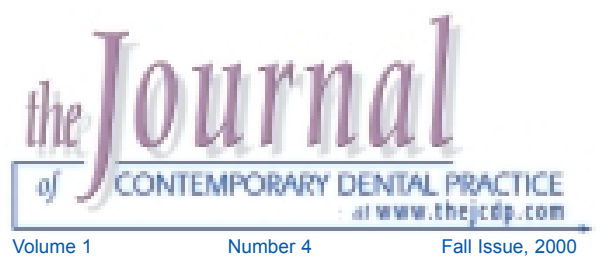

\title{
Four-Handed Dentistry Revisited
}

\section{Betty Ladley Finkbeiner, CDA, RDA, MS}

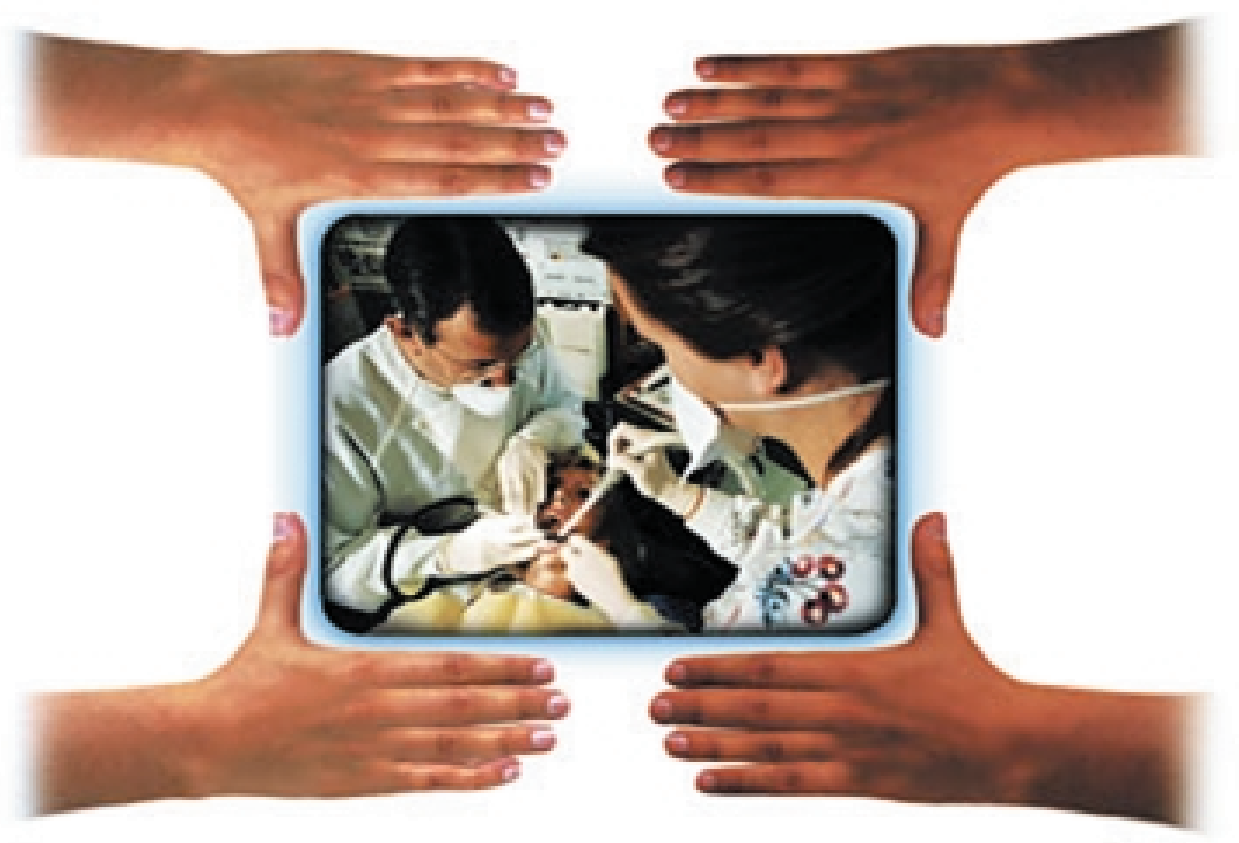

Abstract

A great deal of new technology has been integrated into the modern dental office during the last several decades. However, no product can increase productivity and reduce stress and strain on the dental team as much as using the singular concept of four-handed dentistry. The research of the 1950s is undaunted in its impact on productivity. Combined with the practice of ergonomics in the workplace, this concept must be revisited by the dental profession. The young dentists of the 21 st Century have had minimal exposure to true four-handed dentistry. This article, the first in a series, re-introduces the basic principles of a research-oriented concept.

Keywords: Four-handed dentistry, ergonomics, motion economy, classifications of motion 


\section{Introduction}

This is the first in a series of articles that will appear in future editions of this Journal which will examine the concept of four-handed dentistry. Topics will include the basic principles of fourhanded dentistry, equipment selection, how to increase productivity and decrease stress, as well as provide the dental team with a review of basic clinical skills and suggestions for improving chair side techniques. A final article will look at the application of ergonomic concepts in the dental business office.

This article will focus on the principles of fourhanded dentistry and how these concepts can bring about change in thinking that can increase productivity and reduce stress and strain on the dental team.

The objectives of this article are to:

- identify the basic tenets of four-handed dentistry,

- describe motion economy,

- identify typical zones of activity,

- define dental team responsibilities to ensure effective four-handed dentistry and

- list twenty-five typical excuses for a closed mind

Ask just about any dentist today if he or she practices four-handed dentistry and you will probably hear a resounding "yes." Yet, ask the same dentists if they practice true four-handed dentistry and adhere to principles of motion economy and ergonomic concepts, and the positive answers become less resounding.

Despite the keen interest in the issue of ergonomics in the dental workplace by the Occupational Safety and Health Administration (OSHA), fewer dental schools teach the concepts of four-handed dentistry as part of the curriculum. The result is that many dental graduates learn four-handed dentistry on the job, from formally trained dental assistants or from assistants who themselves have been trained on the job.
Four-handed dentistry is a team concept where highly skilled individuals work together in an ergonomically designed environment to improve productivity of the dental team, improve the quality of care for dental patients while protecting the physical well-being of the operating team. Four-handed dentistry is not simply transferring instruments from one person to another nor is it "hurry-up" dentistry. Rather four-handed dentistry is the way to work smarter, not harder.

\section{Basic Tenets of Four-handed Dentistry}

Some dentists and assistants claim they practice four-handed-dentistry, yet they still suffer the results of physical stress due to the use of inappropriate equipment and techniques that fall short of meeting the basic tenets of the fourhanded dentistry concept. Dentists can still be observed changing their own burs, or twisting and turning to reach equipment on their side of the chair. If the assistant is not in charge of all instrument transfers and the equipment is not within reach of the assistant, true four-handed dentistry is not being practiced.

The concept of four-handed dentistry is not production line dentistry. It is based on a set of criteria that define the conditions under which efficiency can be attained.

\section{To practice true four-handed dentistry, the following criteria must be met:}

1. Equipment must be ergonomically designed to minimize unnecessary motion.

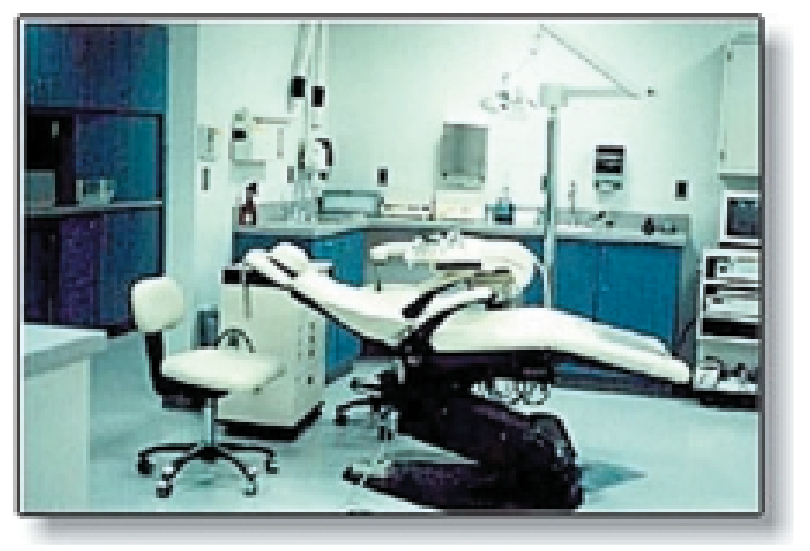


2. The operating team and patient are seated comfortably in ergonomically designed equipment.

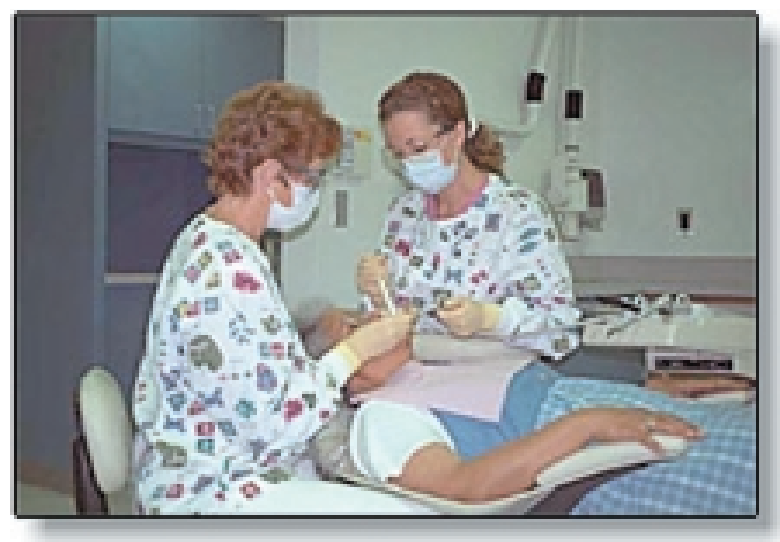

3. Motion economy is practiced.

4. Preset cassettes/trays are utilized.

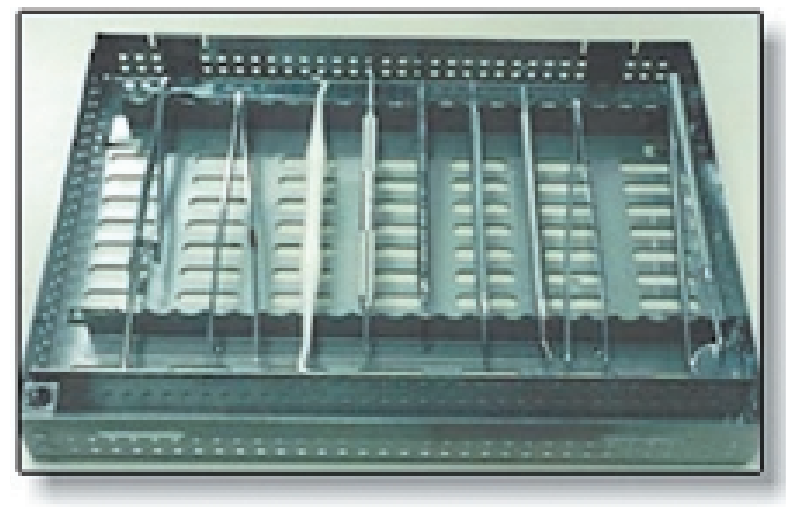

5. The dentist assigns all legally delegable duties to qualified auxiliaries based on the state's guidelines.

6. Patient treatment is planned in advance in a logical sequence.

\section{Principles of Motion Economy}

Motion economy refers to the manner in which human energy can be conserved while performing a task. The objective in all areas of the dental office, clinical, business or laboratory setting, should be to minimize the number and magnitude of motions and conserve energy while working. Ask yourself the following questions:
How many times do you turn your body or reach for an instrument?

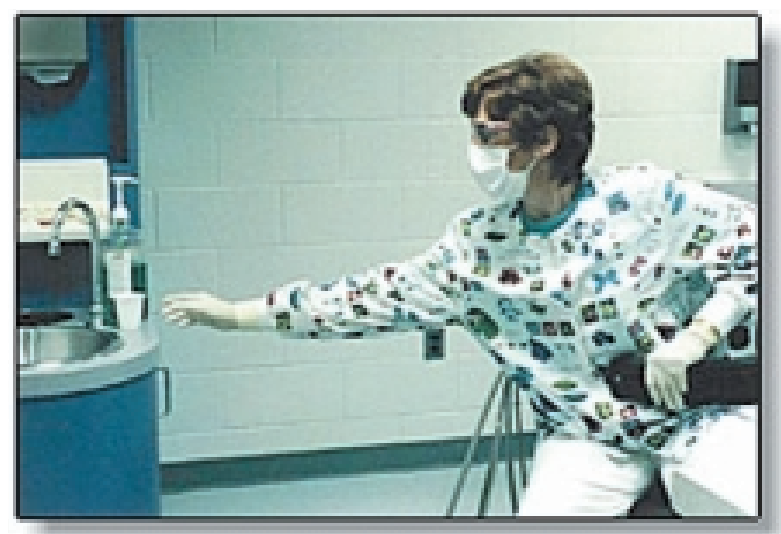

Does the assistant eliminate operator stress by transferring the instruments and materials to the operator?

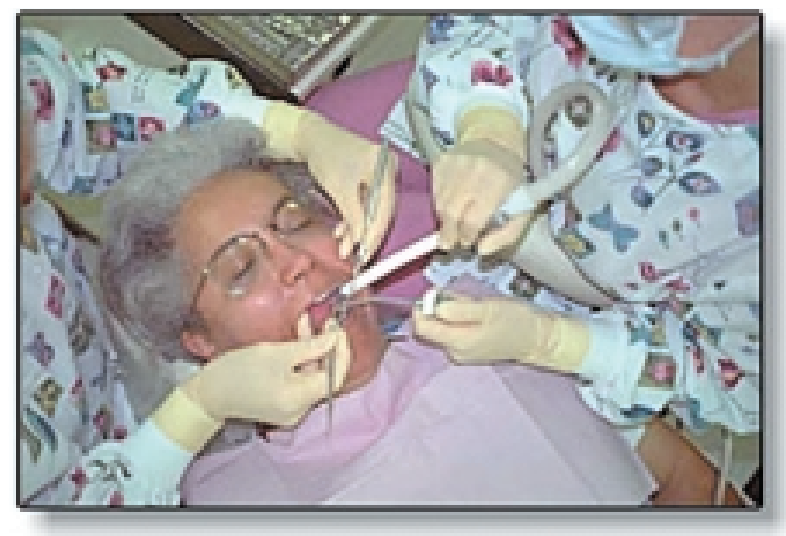

Does the assistant have primary responsibility for transfer of materials and instruments or is the assistant often unoccupied while the dentist reaches for an instrument or changes a bur?

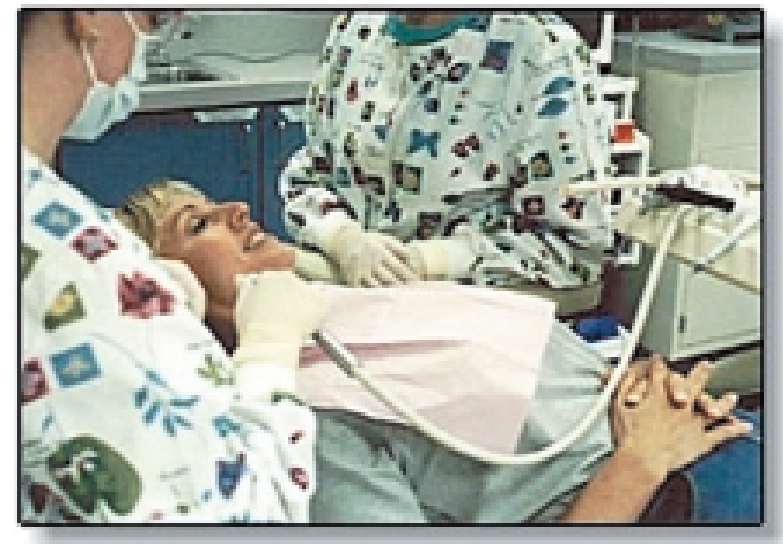


Are the handpieces and instruments within a 21inch radius of the assistant?

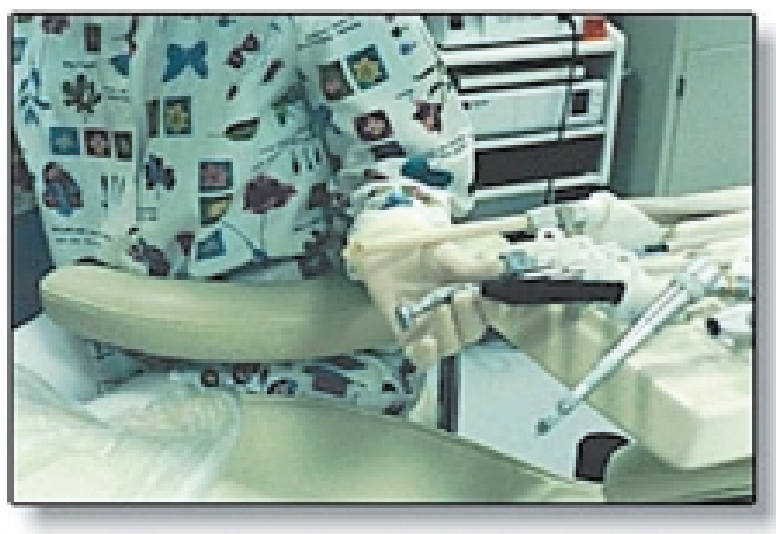

Think about your daily activities and decide how you can modify the routine to reduce motion. Plan ahead to ensure all materials are prepared in advance in a motion effective environment. Perhaps it will be necessary to reorganize a procedure or rearrange materials to achieve this goal.

\section{Classification of Motion}

Motions can be classified into five categories according to the length of the motion as shown below:

Class I Movement of the fingers only as when picking up a cotton roll.

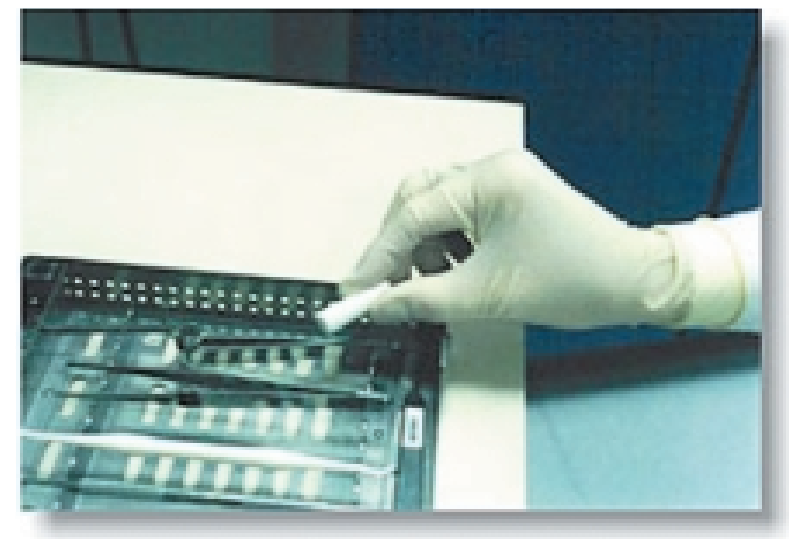

Class II Fingers and wrist motion as used when transferring an instrument to the operator, or manipulating a hand instrument.

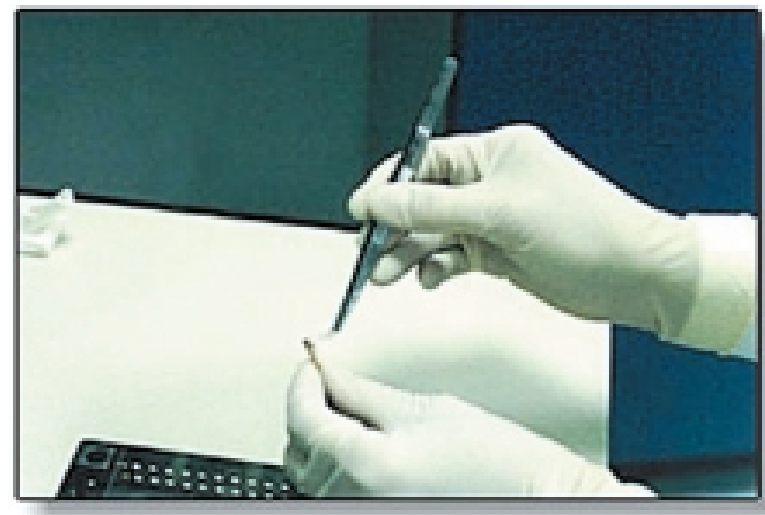

Class III Fingers, wrist, and elbow as when reaching for a handpiece.

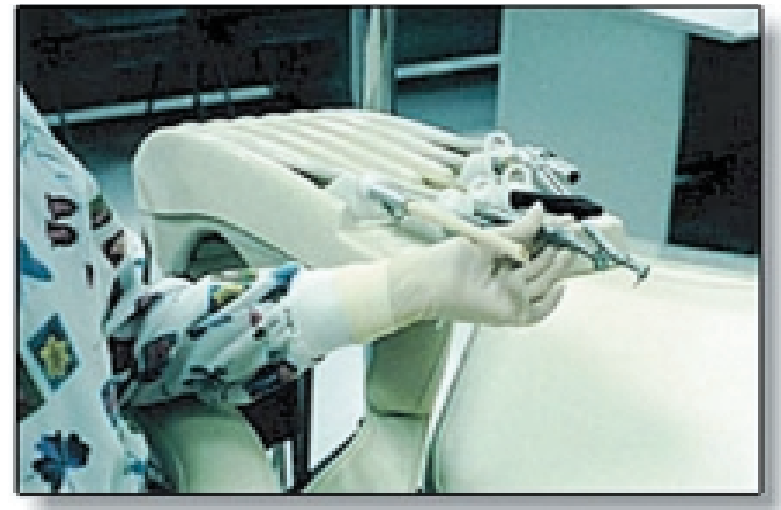

Class IV The entire arm and shoulder as when reaching for supplies away from the work area, or to adjust the operating light.

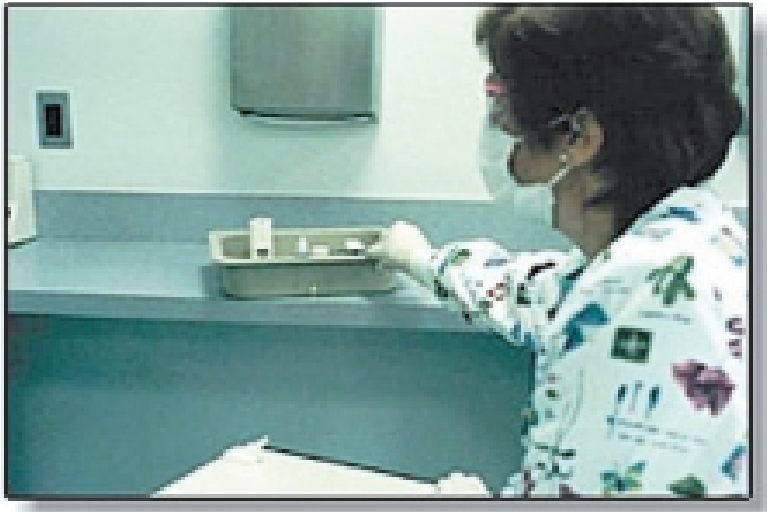


Class V The entire torso as when turning around to reach for equipment from adjacent fixed cabinetry.

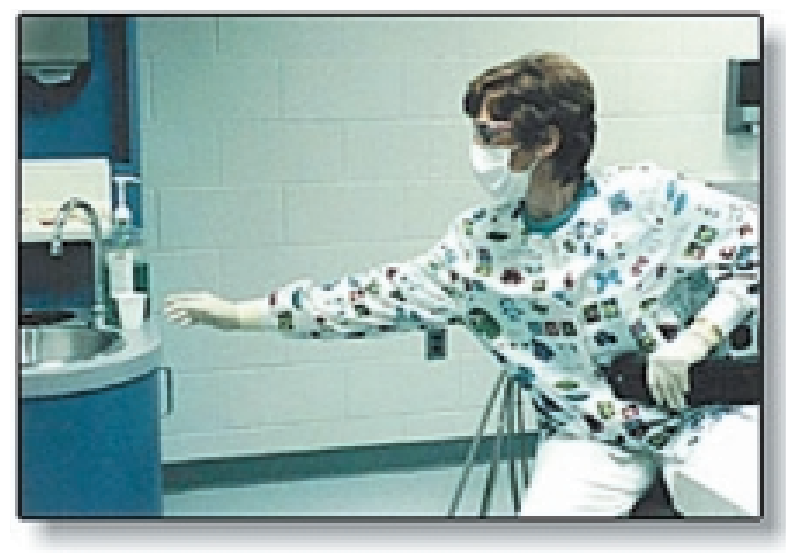

\section{Strategies for Conserving Motion}

Emphasis on modifying motion economy should be given to eliminating Class IV or V motions.

Motion economy should be the primary consideration when purchasing and positioning dental equipment since this concept reduces or eliminates the number and length of motions used during basic treatment procedures. To improve motion economy at chair side, consider the following suggestions:

1. Decrease the number of instruments used for a procedure by maximizing the use of each one for multiple functions.

2. Position the instruments on a preset tray/cassette in the sequence that they will be used. (Figure 13)

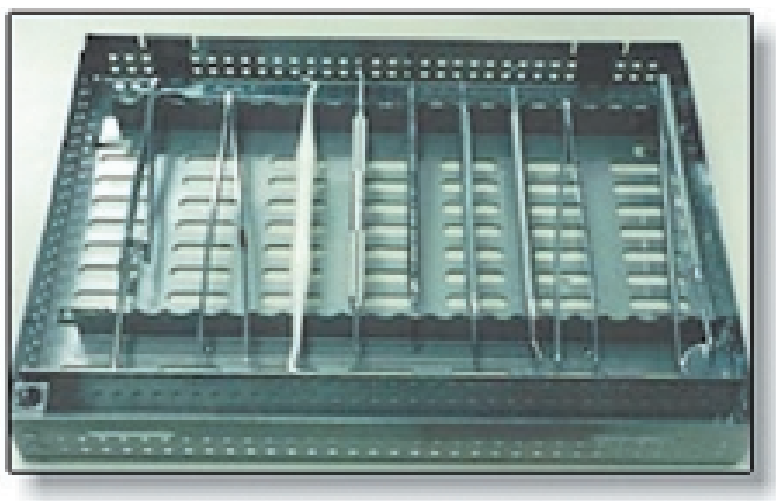

3. Position instruments, materials, and equipment in advance, whenever possible.

4. Place the armamentarium on a mobile cart as close to the patient as possible.

5. Place the patient in a supine position. (Figure 14)

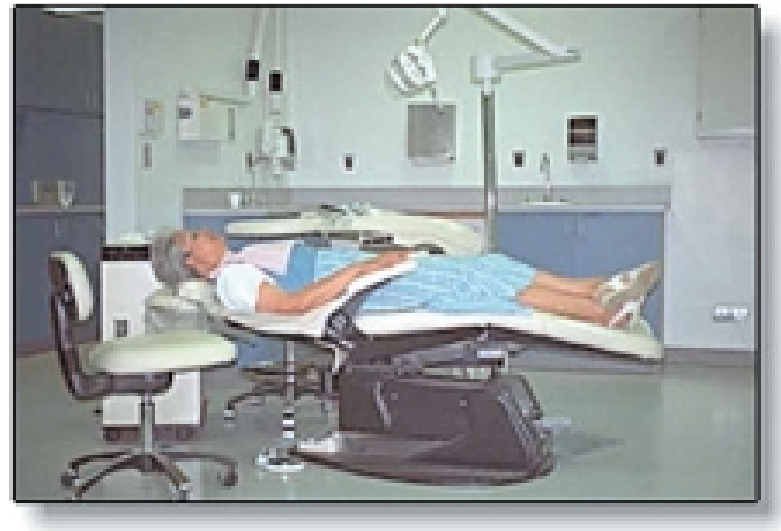

6. Seat the operating team as close to the patient as possible. (Figure 15)

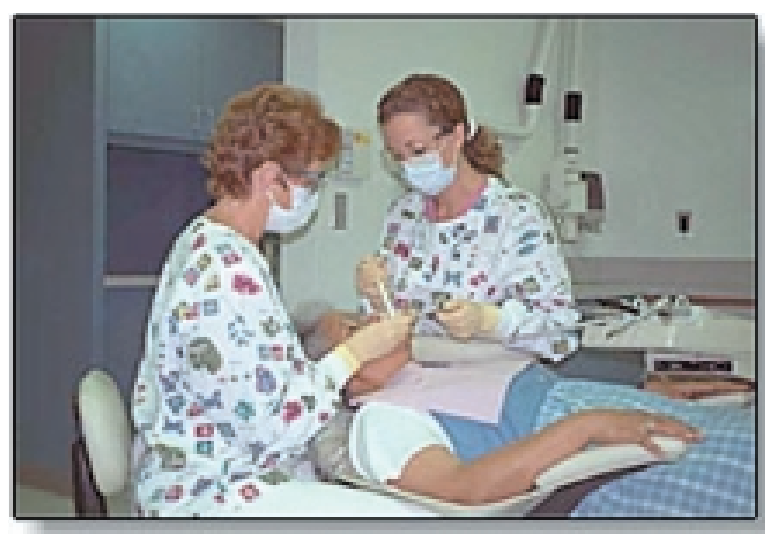


7. Use operating stools that promote good posture and provide back and abdominal support that adjusts vertically and horizontally. (Figure 16)

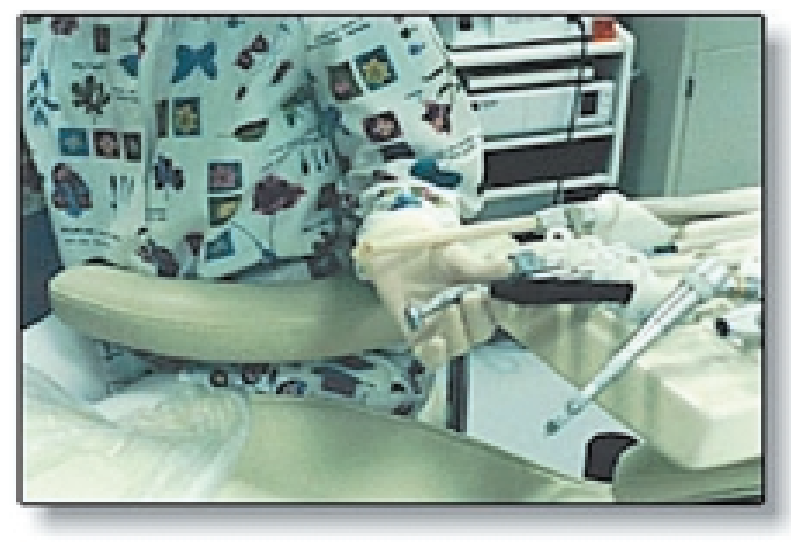

8. Provide work areas that are 1 to 2 inches below the elbow. (Figure 17)

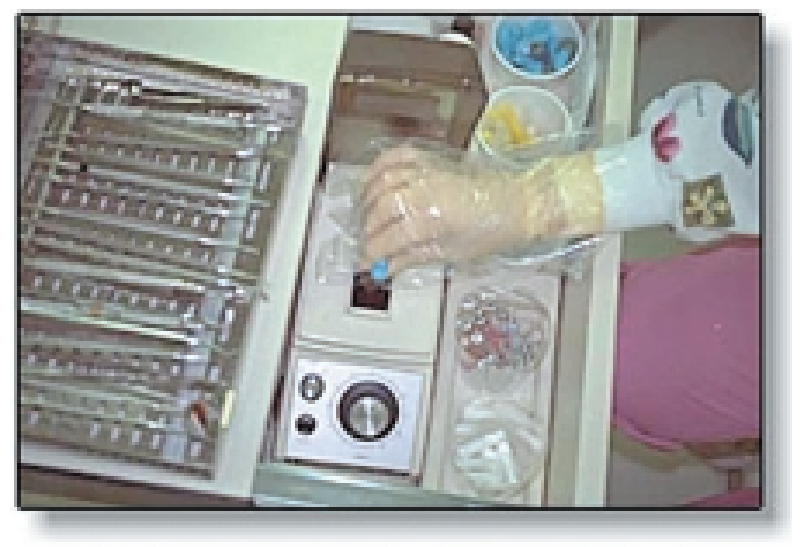

9. Minimize the number of eye movements between the close and brightly lit operating field and more distant objects in the treatment room with lower illumination.

10. Reduce the length and number of motions made by the operator and the dental assistant to accomplish routine and repetitive tasks.

11. Use smooth continuous motions and avoid distracting zigzag movement.

\section{Zones of Activity}

All treatment activity evolves around the patient. Before equipment selection can be considered, the dental team must be aware of special functional spatial relationships around the patient at chair side. The work area around the patient is divided into four "zones of activity." Zones of activity are identified using the patient's face as the face of a clock. The four zones are: the operator's zone, assistant's zone, transfer zone, and static zone. The zones are depicted for a right-handed operator in Figure 18A. The zones are reversed for the left-handed operator. (Figure 18B).

The operator's zone for a right-handed operator extends from 7 to 12 o'clock, the assistant's zone from 2 to 4 o'clock, the instrument transfer zone from 4 to 7 o'clock, and the static zone from 12 to 2 o'clock. The operator changes position dependent upon the dental arch and tooth being treated. The assistant seldom moves much in the zone of activity, but may find it necessary to raise the operating stool when working on the mandibular arch to improve the line of sight into the oral cavity.

These zones are self-explanatory except for the static zone, which is the zone of least activity. Instruments that are infrequently used such as the blood pressure equipment, portable curing light, or the assistant's mobile cabinet when not in use can be stored in this area.

An awareness of the zones of activity is important since they should serve as guides for the dental team in locating equipment, as well as, positioning the patient and operating team to facilitate access to the operative field and improve visibility. The application of this concept will be covered in a later issue of this journal, as will equipment selection, and an in depth look at the ergonomics of dental team positioning. 


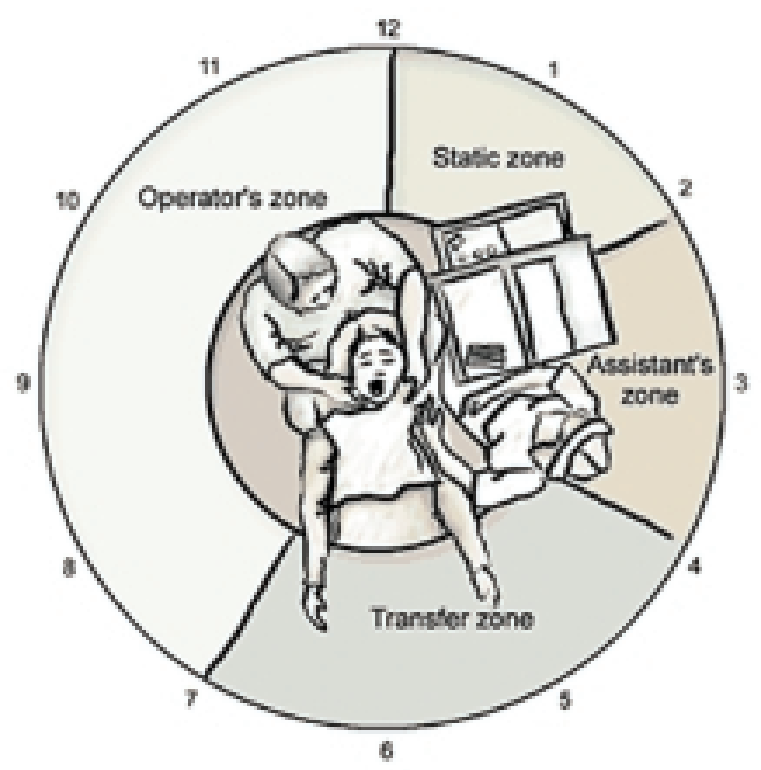

Fig. 18A Zones of Activity for a right-handed dentist.

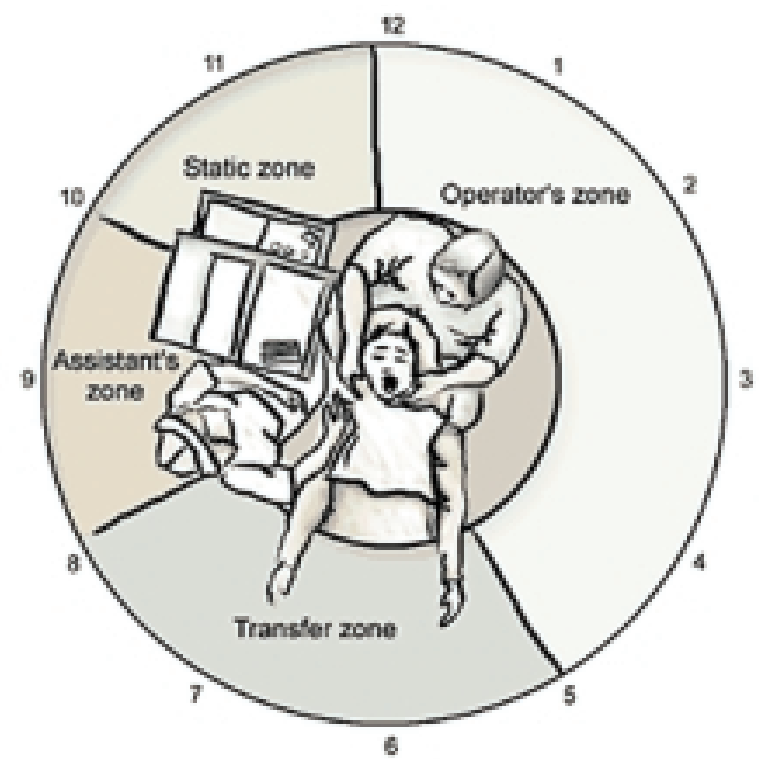

Fig. 18B Zones of Activity for a left-handed dentist.

\section{Strategies to Ensure Effective Four-handed Dentistry}

\section{Teamwork}

To effectively implement the four-handed dentistry concept each member of the dental team must assume individual as well as team responsibilities. The team must be aware of each other's needs, recognize the need to reposition the patient and operating team, as necessary to reduce strain, improve access and visibility, and reduce unnecessary movement by transferring instruments only within the transfer zone.

\section{Strategies for the Operator}

The dentist/operator must develop a standardized routine for basic dental procedures, a non-verbal signal denoting a need to exchange an instrument, and when necessary, give advance distinct verbal direction to communicate a need for a different instrument or material. Additionally, the operator must maintain a working position within the operator's zone, avoid legs interfering with the static or assistant's zone, confine eye focus to the field of operation, and confine hand and arm movement to the transfer zone. To reduce stress and strain the dentist/operator must avoid twisting and turning to reach instruments by relying on the assistant to change burs and to transfer needed instruments, exchange instruments only in the transfer zone, and avoid removing instruments from the preset tray by returning instruments to the assistant.

\section{Strategies for the Dental Assistant}

The clinical assistant must develop a thorough understanding of the procedure, recognize the patient's needs, anticipate the operator's need, and recognize any change in the procedure.

During the procedure the assistant should be seated as close to the patient as possible with legs parallel to the long axis of the patient's body. The assistant must maintain the order of both the instruments and dental materials according to their sequence of use. When transferring a dental instrument to the operator, orient the working end of the instrument so it is pointing up for maxillary functions and down for mandibular functions. The assistant should work with the operator to establish and follow a safe, standardized, and predictable instrument transfer 
protocol. When practical the dental assistant should change burs in the handpiece, maintain a "ready position" for delivery of the handpiece, remove debris from instruments before returning them to the preset tray, and maintain a clean work area at all times.

\section{Twenty-Five Excuses for A Closed Mind Toward the Practice of Ergonomic Concepts in Four-handed Dentistry}

Some of the most common reasons or excuses for not practicing ergonomics in dentistry are listed below. These reasons have been given by dentists of all ages; some with newly designed offices and others with offices that were designed 20 or more years ago.

1. It doesn't seem right.

2. I have more diversion when I do some of the things myself.

3. The assistant says it is too much bother to do more work.

4. I like reaching for my own equipment.

5. It relaxes me to look away from the patient and not concentrate so much.

6. I really haven't had many sick days.

7. It costs too much.

8. It seems so different.

9. I didn't learn it in dental school.

10. My assistant doesn't know how.

11. I like the way I am doing it now.
12. That is how the equipment is designed, so I just work around it.

13. It sounds too scientific.

14. It's too radical a change.

15. I tried it once and didn't like it.

16. The office I bought wasn't set up for it.

17. I don't like to be confined.

18. It sounds like a production line.

19. I'm too laid back.

20. It's not required by OSHA.

21. It won't work in our office.

22. I think we should look into it further before we act.

23. It won't pay for itself.

24. I know a fellow who tried it.

25. We've always done it this way.

\section{Conclusion}

In this brief overview the reader has been exposed to the basic principles of four-handed dentistry. These principles allow the practitioner to work smarter, not harder. When the concepts discussed in this article are applied to the dental team's daily practice, it is likely that the team will identify areas that relate to increased stress and reduced productivity. If so, keep an open mind and begin today to determine how changes can be made to modify your mode of practice to make it more productive and less stressful. 


\section{References}

1. Chasteen, J.E. Four-handed Dentistry in Clinical Practice, St. Louis, C.V. Mosby Company, 1978.

2. Finkbeiner B.L. and Johnson C.J. Comprehensive Dental Assisting: A Clinical Approach, St. Louis, Mosby Year Book, 1995.

3. Finkbeiner B.L. Four-handed Dentistry: A handbook of clinical application and ergonomic concepts, Prentice Hall, New Jersey, 2000.

4. Kilpatrick H.C. Functional Dental Assisting, W.B. Saunders Co., Philadelphia, 1977.

5. Murphy D.C. Ergonomics and the Dental Health Care Worker. Washington, DC American Public Health Association, 1998.

6. University of Alabama. Four-handed dentistry manual, ed 6, Birmingham. 1990.

\section{About the Author}

\section{Betty Ladley Finkbeiner, CDA RDA, MS}

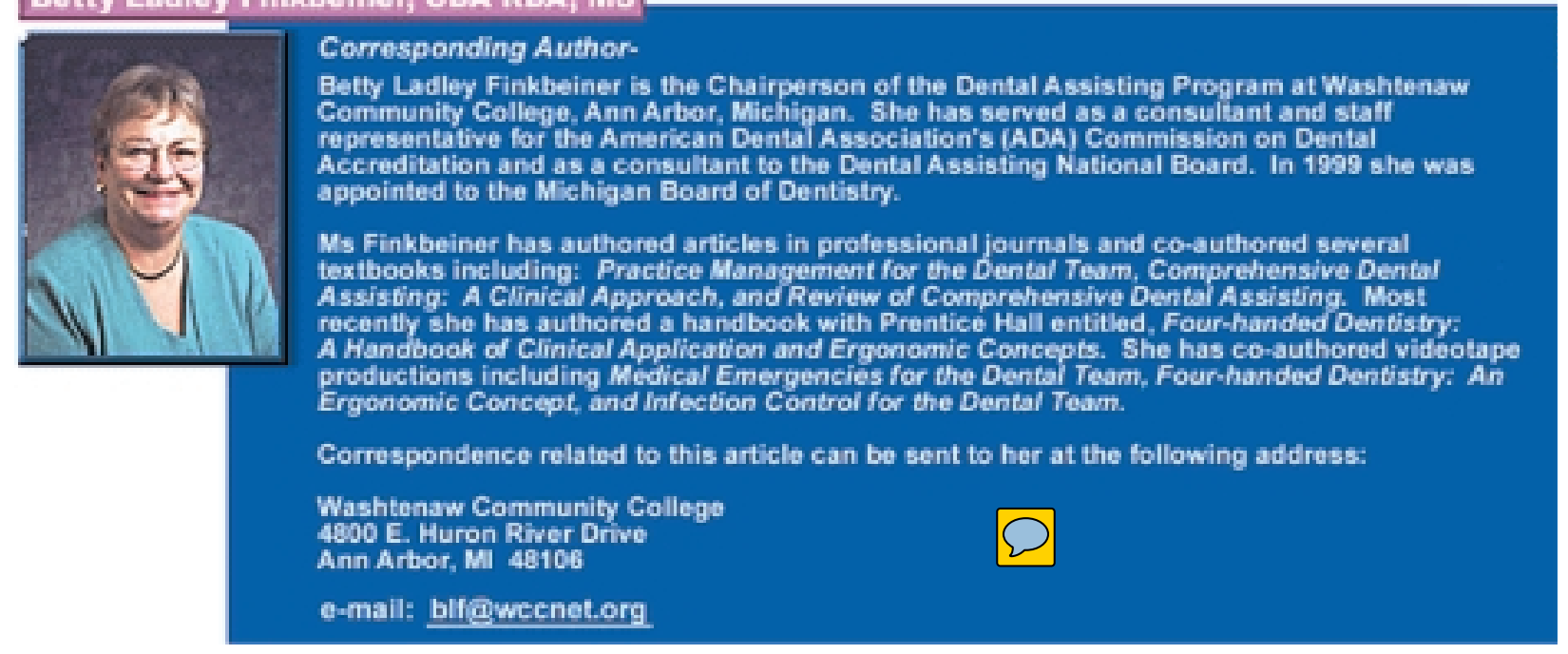

